\title{
Progression of Precancerous Cervical Lesion Predicted by p16 Protein Immunohistochemistry in Rajavithi Hospital
}

\author{
Tipnaree Charoonwatana*, Sathone Boonlikit, Marut Yanaranop
}

\begin{abstract}
Objective: To assess the association of p16 immunohistochemical (IHC) staining in cervical squamous intraepithelial lesions (SIL) and progression of cervical intraepithelial neoplasia (CIN) 1 to CIN2+ or recurrence of CIN2+. Material and Methods: A retrospective cohort study of women with newly diagnosed SIL from colposcopy-directed biopsy at Rajavithi Hospital, 2013-2017. Pathologic specimens were reviewed and submitted to p16-IHC staining. Adjusted hazard ratios (HR) of disease-free interval (DFI) and 95\% confidence intervals (CI) were carried out using the Cox proportional hazard regression model. Results: A total of 187 women was recruited, 91 cases of positive p16-IHC staining and 96 cases of negative staining. With the median follow-up time of 22 months, women with positive p16-IHC had significantly lower 1-year DFI than those with negative p16-IHC (86.8\% vs. 96.6\%, p =0.006). Women with CIN 1 had $22.6 \%$ of positive p16-IHC, while those with CIN2-3 had 86.7\%. From multivariate analysis, the positive p16-IHC and age $>$ 35 years were the significant prognostic factors of progression/recurrent CIN2+ (adjusted HR 5.33, 95\%CI 1.77-16.01, $\mathrm{p}=0.003$; and adjusted HR 5.80,95\% CI 1.34-25.08, $\mathrm{p}=0.019$, respectively). From subgroup analysis, the positive p16-IHC was the significant prognostic factor in women with initial CIN1 (HR 5.29, 95\%CI 1.18-23.76, p = 0.030), but was not associated with prognosis in women with initial CIN 2-3 (HR 2.13, 95\% $0.28-16.38, p=0.468)$. Conclusion: Overexpression of p16 protein has the prognostic significance of SIL. Using p16-IHC may help stratify patients as low-risk and high-risk groups to progression/recurrence CIN2+.
\end{abstract}

Keywords: p16 protein immunohistochemistry- cervical intraepithelial neoplasia- precancerous cervical lesion

Asian Pac J Cancer Prev, 20 (6), 1809-1815

\section{Introduction}

Cervical cancer is the fourth most frequent cancer in women worldwide(Bruni et al., 2017) which remains a leading cause of cancer-related death for women in developing countries. As known, it is a well-controlled disease in industrialized countries because of Papanicolaou (PAP) test and effective screening program implementation. (Sherris et al., 2001; Catarino et al., 2015; Torre et al., 2017) Disease prevalence is decreasing in those population. Further attention should be paid in specific screening algorithm to enhance the efficacy of screening protocols. It is well known that cervical cancer is preceded by high grade intraepithelial lesion (HSIL) which follows persistent human papillomavirus (HPV) infection.

In fact, active transcription of HPV oncogene can be directly detected by E6/E7 viral mRNA transcripts and indirectly detected by accumulation of the host protein p16 in the cell. It has been widely reported that p16 expression is affected by the high risk HPV E7 protein and its up-regulation increasing severity of cervical lesions. (Giarrè et al., 2001; Li et al., 2011) Protein p16, a tumor suppressor from the Ink4 family, encoded by CDKN2A gene (9p21.3) prevents progression into $\mathrm{S}$ phase of cell cycle by inhibits cyclin $\mathrm{D}$ dependent protein kinases (CDK4 and CDK6) therefore maintaining retinoblastoma protein $(\mathrm{pRb})$ in its hypophosphorylated state which prevents its dissociation from E2F transcription factor. Integration of viral E7 oncoprotein integrate into host genome leads to inactivation of $\mathrm{pRb}$ and overexpression of $\mathrm{p} 16$, therefore $\mathrm{p} 16$ protein immunohistochemistry (p16 IHC) is surrogate marker of high risk HPV infection. (Sano et al., 1998; Klaes et al., 2001; Negri et al., 2004; Queriroz et al., 2006; Iaconis et al., 2007)

The main objective of the present study was to assess the association of the overexpression of p16 IHC and progression of cervical intraepithelial neoplasia (CIN) 1 to CIN2 or worse (CIN2+) or recurrence of CIN2+ after treatment during follow up in women who had a colposcopy-directed biopsy (CDB).

\section{Materials and Methods}

A retrospective cohort study, the women who newly diagnosed SIL from CDB at the Department of Obstetrics and Gynecology of Rajavithi Hospital, Bangkok from January 2013 to June 2017, all the patients showing histologically confirmed precancerous cervical lesion at 
an initial evaluation and followed up at least 1 year from the date of histologic diagnosis by CBD to the last visit were included in this study.

\section{Study design and Population}

Patients whom diagnosed precancerous cervical lesion from CBD including HPV CIN1 CIN2 CIN3 were recruited, $\mathrm{H}$ and $\mathrm{E}$ slide were recut and reviewed with blinding previous diagnosis. Then p16 IHC were stained and interpreted by same pathologist in different time. Positive staining was defined as "block" staining: strong abnormal nuclear and cytoplasmic expression in a continuous segment of cells (at least 10 - 20 cells); in squamous epithelium, block positivity needs to involve basal and parabasal layers. Cytoplasmic only staining, diffuse blush / weak intensity staining / other focal / patchy patterns should be considered negative as. (Darragh et al., 2012; Kurman et al., 2014) The exclusion criteria were previous history of SIL, cervical cancer and absent paraffin block. Patients who underwent see \& treat strategy were not analyzed.

\section{Follow-up and Outcome}

All patients underwent standard treatment during followed up, when abnormal cytology screening (ASCUS or worse) was presented, cervical tissue was confirmed by CDB. After diagnosis of precancerous cervical lesion, patients with low-grade lesions (HPV or CIN1) were observed every 6 months by conventional pap test or Liquid-based cytology at each follow-up while patients with high-grade lesion had intervention as standard protocol either Loop Electrosurgical Excision Procedure (LEEP) or local ablation then patients were followed up with cytology every 6 months. If there was any abnormality, CDB was done to confirm histologic diagnosis but in case of normal colposcopy, CBD was not performed. The outcome was measured by disease free interval(DFI) which was the time from diagnosis to progression to $\mathrm{CIN} 2+$ in case $\mathrm{HPV} / \mathrm{CIN} 1$ or the time from diagnosis to recurrence to CIN2+ in case CIN2-3 after treatment (Health, 2014).

\section{Statistical Analysis}

The study sample size was estimated based on the formula for two independent proportion formula of Bernard (2000)??? with 1:1 ratio, a proportion in group 1 of 0.250 and group 2 of 0.029 from study of Razmpoosh et al., (2014) were incorporated into the calculation. All data was analyzed using STATA program fifteenth version, the descriptive analysis were described by mean, $\mathrm{SD}$, median, range for numerical data and using proportion, percentage for categorical data. The inferential analysis was compared by Student t-test, Chi-square test, Fisher exact test. The $95 \%$ confidence intervals (CI) were carried out using the Cox proportional hazard regression model. A value of $\mathrm{p}$ $<0.05$ was considered statistically significant.

\section{Results}

The 263 women who newly diagnosed SIL were recruited, then 76 women who absented paraffin block were excluded. Finally, 187 women were analyzed by p16 IHC staining. Ninety-one women were positive for p16 IHC staining and 96 women were negative for $\mathrm{p} 16$ IHC staining.

The baseline characteristics including age parity smoking and immunocompromised status were balance but initial histology and treatment were statistically significant difference as summarized in table1. The mean age of participants was 40.82 and 42.31 years in positive p16 and negative p16 staining respectively, most of them were multi-parity no smoking and no underlying immunocompromised status. Mostly, LSIL were negative p16 about $77.7 \%$ and progressed to CIN2+ only $3.6 \%$

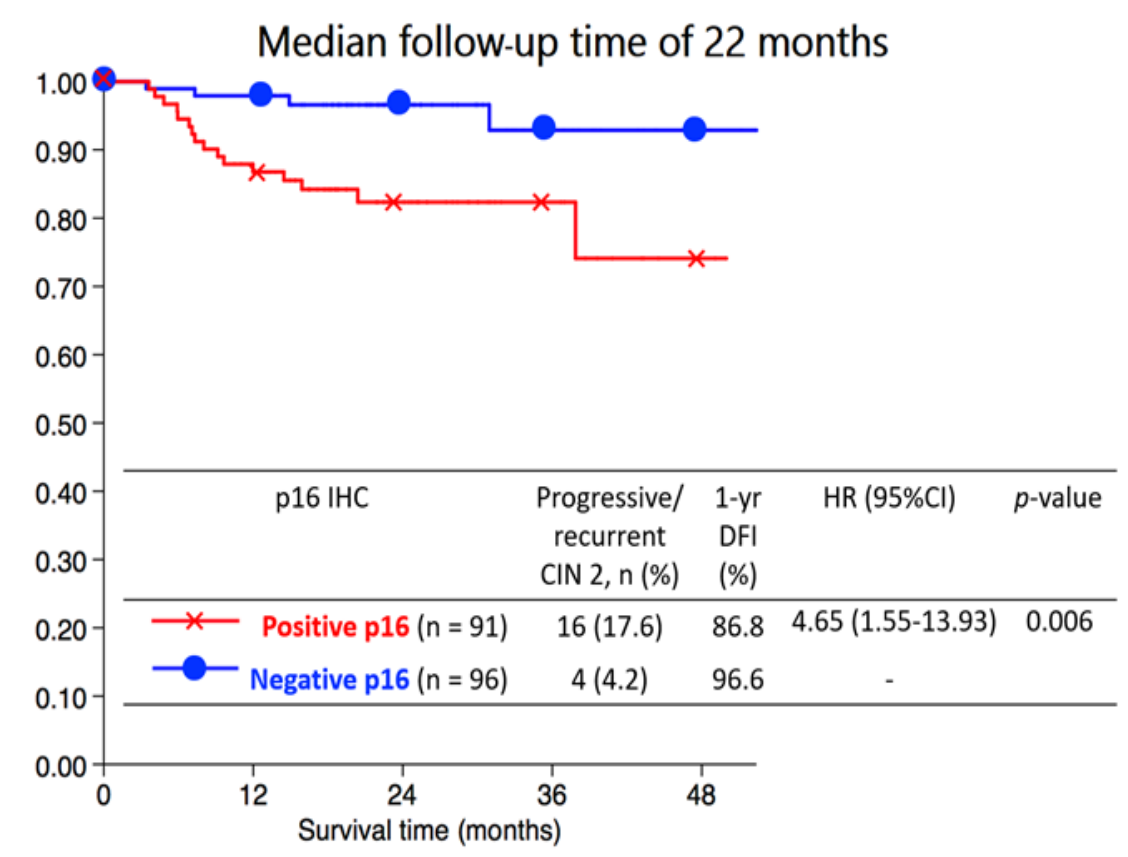

Figure 1. The Kaplan Meier Curve for 1 Year DFI of p16 IHC and Progressive/recurrent CIN2+ 


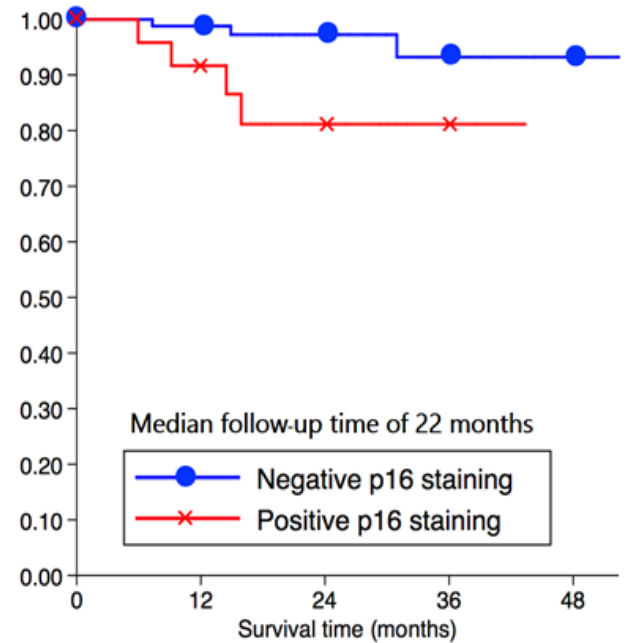

(a)

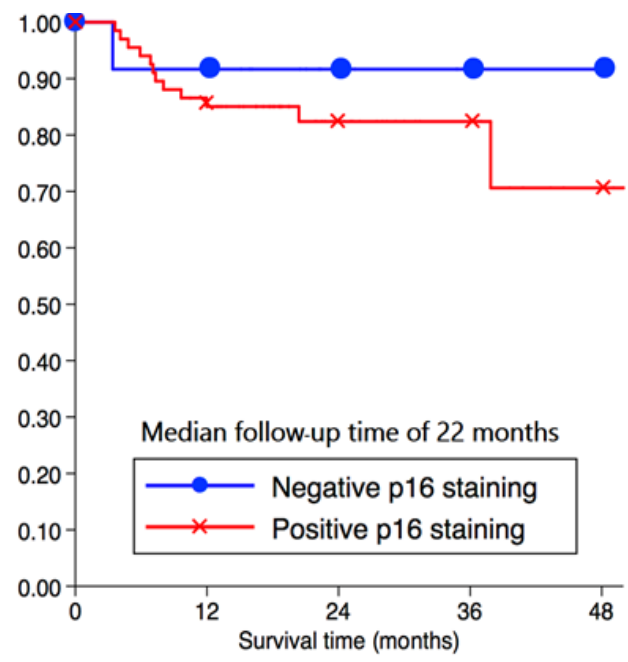

(b)

Figure 2. The Kaplan Meier Curve for 1 Year DFI of p16 IHC and Progressive CIN2+ in LSIL (a) and HSIL (b)

Table 1. The Baseline Characteristics

\begin{tabular}{|c|c|c|c|c|c|}
\hline Characteristics & \multicolumn{2}{|c|}{$\begin{array}{c}\text { Positive p16 } \\
\mathrm{n}=91(\%)\end{array}$} & \multicolumn{2}{|c|}{$\begin{array}{c}\text { Negative p16 } \\
\mathrm{n}=96(\%)\end{array}$} & \multirow{2}{*}{$\begin{array}{c}\mathrm{p} \text {-value } \\
0.39\end{array}$} \\
\hline Age (yrs.), mean (SD) $\dagger$ & 40.82 & $(12.5)$ & 42.31 & $(11.1)$ & \\
\hline \multicolumn{6}{|l|}{ Parity $t$} \\
\hline Nulliparity & 17 & $(19.5)$ & 15 & (16) & \multirow[t]{2}{*}{0.563} \\
\hline Multiparity & 74 & $(80.5)$ & 79 & $(84)$ & \\
\hline Immunocompromised status $\ddagger$ & 7 & (7.7) & 10 & $(10.4)$ & 0.517 \\
\hline Smoking § & 1 & $(1.1)$ & 2 & $(2.1)$ & 0.999 \\
\hline \multicolumn{6}{|l|}{ Histology $\ddagger$} \\
\hline LSIL & 24 & $(22.6)$ & 84 & $(77.4)$ & \multirow[t]{2}{*}{$<0.001^{*}$} \\
\hline HSIL & 67 & $(86.7)$ & 12 & $(13.3)$ & \\
\hline \multicolumn{6}{|l|}{ Treatment $\ddagger$} \\
\hline None & 33 & $(36.3)$ & 72 & $(75)$ & \multirow[t]{3}{*}{$<0.001^{*}$} \\
\hline LEEP & 55 & $(60.4)$ & 24 & $(25)$ & \\
\hline Hysterectomy & 3 & $(3.3)$ & 0 & 0 & \\
\hline
\end{tabular}

HSIL, high-grade squamous intraepithelial lesion; LEEP, loop electrosurgical excision procedure; LSIL, low-grade squamous intraepithelial lesion; p16, p16 protein-immunohistochemistry; SD, standard deviation; *, Significance at p-value less than 0.05 ; $†$ Student t-test, $\$$, Chi-square test; $\S$, Fisher-exact test.

while the progression of positive p16 LSIL was $16.7 \%$ In contrast, HSIL found $84.8 \%$ of HSIL had positive p 16 and recurrence were $17.9 \%$ as shown in Table 2.

The Kaplan Meier curve for 1-year DFI showed that women who had positive p16 had significantly lower than negative $\mathrm{p} 16(86.8 \%$ vs. $96.6 \% ; \mathrm{p}=0.003)$ with the median follow up time of 22 months as Figure 1. The univariate analysis of variable associated progression or recurrence to CIN2+ revealed that prognostic factors were age, initial histology, and p16 staining. After performing the multivariate analysis, it showed the p16 IHC and age $>35$ years were significant prognostic factors for poor outcomes after adjust other factors (Table 3 ).

The Subgroup analysis of Prognostic Factors Related to Progressive CIN2+, positive p16 was important prognostic factor with significant difference in women diagnosed with low-grade lesion (Table 4). 1-year DFI in those with positive p16 IHC $(91.7 \%)$ was lower than those with negtive p16 (98.8\%) with significant difference, which was confirmed by the Kaplan Meier curve for 1-year

Table 2. Progression of Precancerous Cervical Lesion Associated with p16 IHC Staining

\begin{tabular}{llcc}
\hline & & Total $(\%)$ & Progressive/recurrent CIN2+ $(\%)$ \\
\hline LSIL & Negative p16 IHC & $84(77.7)$ & $3(3.6)$ \\
$(\mathrm{N}=108)$ & Positive p16 IHC & $24(22.2)$ & $4(16.7)$ \\
HSIL & Negative p16 IHC & $12(15.2)$ & $1(8.3)$ \\
$(\mathrm{N}=79)$ & Positive p16 IHC & $67(84.8)$ & $12(17.9)$ \\
\hline
\end{tabular}

CIN2+, cervical intraepithelial neoplasia 2 or worse; p16 IHC, p16 protein-immunohistochemistry staining 


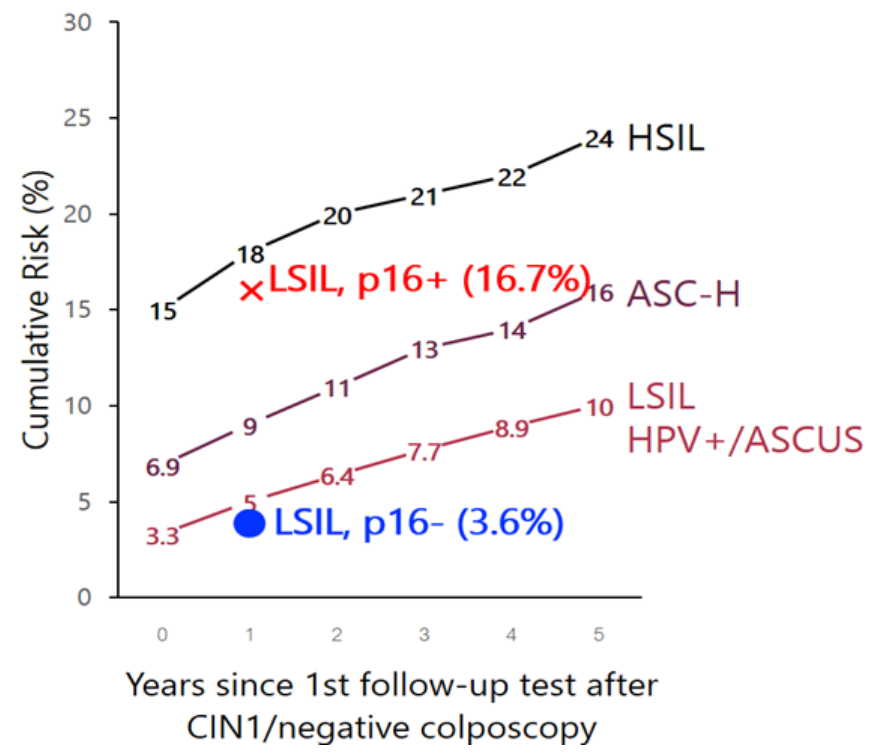

Figure 3. Benchmark of Cumulative Risk of CIN2+ Following CIN1/Negative Colposcopy Given Antecedent HSIL+, ASC-H, AGC, and LSIL/HPV+/ASC-US, among Women Aged 25 and Older (Katki et al, 2013). The progressive risk of LSIL with p16 staining positive (cross) and negative (dot) from this study.

Table 3. Factor Associated with Progressive or Recurrent to CIN2 or Worse

\begin{tabular}{|c|c|c|c|c|c|c|c|c|c|}
\hline \multirow{2}{*}{$\begin{array}{l}\text { Variables } \\
\text { Age }\end{array}$} & \multirow[t]{2}{*}{$\mathrm{N}$} & \multicolumn{2}{|c|}{$\begin{array}{l}\text { Progressive/ } \\
\text { recurrent } \\
\mathrm{CIN} 2+(\%)\end{array}$} & \multirow[t]{2}{*}{ Crude HR } & \multirow[t]{2}{*}{$95 \% \mathrm{CI}$} & \multirow[t]{2}{*}{ P-value } & \multirow[t]{2}{*}{ Adjusted HR } & \multirow[t]{2}{*}{$95 \% \mathrm{CI}$} & \multirow[t]{2}{*}{ P-value } \\
\hline & & & & & & & & & \\
\hline$<35$ yrs & 62 & 2 & $(3.2)$ & & & & & & \\
\hline$>35 \mathrm{yrs}$ & 125 & 18 & (14.4) & 4.86 & $(1.13-20.97)$ & $0.034 *$ & 5.8 & $(1.34-25.08)$ & $0.019^{*}$ \\
\hline \multicolumn{10}{|l|}{ Parity } \\
\hline Nulliparity & 33 & 3 & $(9.1)$ & & & & & & \\
\hline Multiparity & 154 & 17 & (11) & 1.41 & $(0.41-4.84)$ & 0.588 & & & \\
\hline \multicolumn{10}{|l|}{ HIV infection } \\
\hline No & 170 & 20 & (11.8) & & & & & & \\
\hline Yes & 17 & 0 & 0 & & & & & & \\
\hline \multicolumn{10}{|l|}{ Smoking } \\
\hline No & 184 & 20 & (10.9) & & & & & & \\
\hline Yes & 3 & 0 & 0 & & & & & & \\
\hline \multicolumn{10}{|l|}{ Initial histology } \\
\hline LSIL & 108 & 7 & $(6.5)$ & & & & & & \\
\hline HSIL & 79 & 13 & (16.5) & 2.8 & $(1.12-7.03)$ & $0.028 *$ & & & \\
\hline \multicolumn{10}{|l|}{ p16 IHC staining } \\
\hline Negative & 96 & 4 & $(4.2)$ & & & & & & \\
\hline Positive & 91 & 16 & (17.6) & 4.65 & $(1.55-13.93)$ & $0.006^{*}$ & 5.33 & $(1.77-16.01)$ & $0.003 *$ \\
\hline \multicolumn{10}{|l|}{ Treatment } \\
\hline Observation & 105 & 10 & $(9.5)$ & & & & & & \\
\hline LEEP & 79 & 9 & (11.4) & 1.27 & $(0.52-3.13)$ & 0.6 & & & \\
\hline Hysterectomy & 3 & 1 & (33.3) & 4.42 & $(0.56-34.73)$ & 0.158 & & & \\
\hline
\end{tabular}

CIN2+, cervical intraepithelial neoplasia 2 or worse; HSIL, high-grade squamous intraepithelial lesion; LEEP, loop electrosurgical excision procedure; LSIL, low-grade squamous intraepithelial lesion; p16 IHC, p16 protein-immunohistochemistry; * Significance at p-value less than 0.05 ; $\dagger$, Student t-test; $\$$, Chi-square test, §Fisher-exact test

DFI of p16 IHC and progressive CIN2+ in Figure 2a. However, in women diagnosed with high-grade lesion, positive p16 was not significantly associated recurrence to CIN2+ after treatment (Table 5) which shown in the
Kaplan Meier curve of DFI in Figure 2b. 
Table 4. Factor Associated with Progressive to CIN2 or Worse in LSIL

\begin{tabular}{|c|c|c|c|c|c|c|}
\hline Variables & $\mathrm{N}$ & \multicolumn{2}{|c|}{ Progressive CIN2+ (\%) } & Crude HR & $95 \% \mathrm{CI}$ & P-value \\
\hline \multicolumn{7}{|l|}{ Age } \\
\hline$<35$ yrs. & 32 & 2 & $(6.25)$ & & & \\
\hline$>35$ yrs. & 72 & 5 & $(6.94)$ & 1.4 & $(0.27-7.26)$ & 0.684 \\
\hline \multicolumn{7}{|l|}{ Parity } \\
\hline Nulliparity & 21 & 1 & $(4.76)$ & & & \\
\hline Multiparity & 87 & 6 & $(6.9)$ & 1.54 & $(0.18-12.80)$ & 0.691 \\
\hline \multicolumn{7}{|l|}{ HIV infection } \\
\hline No & 97 & 7 & $(7.22)$ & & & \\
\hline Yes & 11 & 0 & & & & \\
\hline \multicolumn{7}{|l|}{ Smoking } \\
\hline No & 107 & 7 & $(6.54)$ & & & \\
\hline Yes & 1 & 0 & & & & \\
\hline \multicolumn{7}{|l|}{ p16 IHC staining } \\
\hline Negative & 84 & 3 & $(3.57)$ & & & \\
\hline Positive & 24 & 4 & $(16.67)$ & 5.29 & $(1.18-23.76)$ & $0.030 *$ \\
\hline \multicolumn{7}{|l|}{ Treatment } \\
\hline Observation & 93 & 7 & $(7.53)$ & & & \\
\hline LEEP & 15 & 0 & & & & \\
\hline
\end{tabular}

\section{Discussion}

In the present study, SIL with positive p16 staining was likely progressed or recurred to CIN2+ with significant difference. Therefore, it is possible that p16 overexpression might be used as a predictor among the patients who diagnosed with SIL. Low-grade lesion with positive p16 staining was likely to progressed about 5.29 times compared to those with negative p16 staining. From previous studies, approximately $10-25 \%$ of CIN1 with positive p16 staining progressed to CIN2+ (Table 6) which were similar to our study. However, the results

Table 5. Factor Associated with Recurrent to CIN2 or Worse in HSIL

\begin{tabular}{|c|c|c|c|c|c|c|}
\hline \multirow{2}{*}{$\begin{array}{l}\text { Variables } \\
\text { Age }\end{array}$} & \multirow[t]{2}{*}{$\mathrm{N}$} & \multicolumn{2}{|c|}{ Recurrent CIN2+ (\%) } & \multirow[t]{2}{*}{ Crude HR } & \multirow[t]{2}{*}{$95 \% \mathrm{CI}$} & \multirow[t]{2}{*}{ P-value } \\
\hline & & & & & & \\
\hline$<50$ yrs & 58 & 9 & $(15.51)$ & & & \\
\hline$>50$ yrs. & 21 & 4 & (19.05) & 1.32 & $(0.41-4.29)$ & 0.644 \\
\hline \multicolumn{7}{|l|}{ Parity } \\
\hline Nulliparity & 12 & 2 & (16.67) & & & \\
\hline Multiparity & 67 & 11 & $(16.42)$ & 1.29 & $(0.27-6.09)$ & 0.743 \\
\hline \multicolumn{7}{|l|}{ HIV infection } \\
\hline No & 73 & 13 & $(17.81)$ & & & \\
\hline Yes & 6 & 0 & & & & \\
\hline \multicolumn{7}{|l|}{ Smoking } \\
\hline No & 77 & 13 & $(16.88)$ & & & \\
\hline Yes & 2 & 0 & & & & \\
\hline \multicolumn{7}{|l|}{ p16 IHC staining } \\
\hline Negative & 12 & 1 & $(8.33)$ & & & \\
\hline Positive & 67 & 12 & $(17.91)$ & 2.13 & $(0.28-16.38)$ & 0.468 \\
\hline \multicolumn{7}{|l|}{ Treatment } \\
\hline Observation & 12 & 3 & $(25)$ & & & \\
\hline LEEP & 64 & 9 & $(14.06)$ & 0.64 & $(0.17-2.41)$ & 0.511 \\
\hline Hysterectomy & 3 & 1 & $(33.33)$ & 1.84 & $(0.19-18.20)$ & 0.601 \\
\hline
\end{tabular}

CIN2+, cervical intraepithelial neoplasia 2 or worse; LEEP, loop electrosurgical excision procedure; p16 IHC, p16 protein-immunohistochemistry *Significance at p-value less than 0.05 
Table 6. Comparation of Previous Studies

\begin{tabular}{|c|c|c|c|c|c|c|}
\hline Author (yr) & $\mathrm{N}$ & Baseline & p16 IHC (\%) & F/U time (mo.) & Progress CIN2+ & Results \\
\hline Razmpoosh (2014)(15) & 64 & CIN1 & 27.4 & $>6$ & $25.00 \%$ & $\mathrm{p}=0.002$ \\
\hline Liao $(2014)(16)$ & 171 & $\begin{array}{c}\text { Normal } \\
\text { CIN1 }\end{array}$ & 42.7 & 24 & $10.70 \%$ & RR 8.25 (1.02-66.62) \\
\hline Pacchiarotti (2014)(17) & 124 & $\begin{array}{c}\text { Normal } \\
\text { CIN1 }\end{array}$ & 50.8 & 24 & $10.30 \%$ & RR 5.20 (0.60-47.50) \\
\hline Sagasta (2016)(18) & 416 & $\begin{array}{l}\text { HPV } \\
\text { CIN1 }\end{array}$ & 11.3 & 28 & $22.40 \%$ & HR $1.6(0.9-2.6)$ \\
\hline This study & 108 & $\begin{array}{l}\text { HPV } \\
\text { CIN1 }\end{array}$ & 22.2 & 22 & $16.70 \%$ & HR 5.29 (1.18-23.76) \\
\hline
\end{tabular}

from those studies were inconclusive. The statistical difference was obvious in 2 studies (Liao et al., 2014; Razmpoosh et al., 2014) whereas not statistically different in another 2 studies (Pacchiarotti et al., 2014; Sagasta et al., 2016). In the present study, high-grade lesion with positive p16 staining had recurrence to CIN2+ about 2.13 times than those with negative p16 staining but it was not significantly different. It is well known that status of LEEP's margin predicts the outcome of treatment, women who have positive excisional margin trend to recur more than those who have negative margin. In the present study, the proportion of women who have positive margin was not identified therefore we do not know exactly how many patients at risk for recurrence in our population. If p16 test is performed in particular group such as women with positive margin, it may be useful and further study in this area is needed.

Base on the concept of equal management of equal risk theory (Katki et al., 2013) in Figure 5, progressive risk of LSIL with negative p16 staining (dot) from the present study is lower than benchmark of Cumulative risk of CIN2+ at 1 year after CIN1/ negative colposcopy by antecedent LSIL / positive HPV DNA ASCUS. These patient might be reassured for follow up without treatment by cotesting at 1 year later followed by ASCCP guideline (Massad et al., 2013). While the progressive risk of LSIL with positive p16 staining (cross) is higher than benchmark (Katki et al., 2013) therefore these patient may benefit more from excision procedure but further studies in larger population is required to confirm these findings.

The important strength of the present study is all slides were histologically reviewed for decrease selective bias and the p16 IHC staining and $\mathrm{H}$ and $\mathrm{E}$ slides were interpreted by the same pathologist in different time and blinding of result. The present study has some limitation. First, the retrospective study leads to incomplete data collected and information bias. Second, a significant loss of paraffin blocks might render the data not representing the actual population.

In conclusion, the overexpression of $\mathrm{p} 16$ protein was the significant prognostic factor of SIL. By using the p16 IHC may help stratify patients as low-risk and high-risk groups to predict progression or recurrence CIN2+.

\section{Funding Statement}

The present study was supported by the research fund from Rajavithi Hospital, Department of medical services.

\section{Conflict of interest}

The authors declare that they have no conflict of interest.

\section{Acknowledgments}

The present study was supported by the Colposcopic Clinic, Department of Obstetrics and Gynecology, Rajavithi Hospital; Immunohistochemistry Division, Department of Pathology, Rajavithi Hospital.

\section{References}

Catarino R, Petignat P, Dongui G, et al (2015). Cervical cancer screening in developing countries at a crossroad. Emerging technologies and policy choices. World J Clin Oncol, 6, 281-90.

Darragh TM, Colgan TJ, Cox JT, et al (2012). The lower anogenital squamous terminology standardization project for HPV-associated lesions: background and consensus recommendations from the College of American Pathologists and the American Society for Colposcopy and Cervical Pathology. Arch Pathol Lab Med, 136, 1266-97.

Giarrè M, Caldeira S, Malanchi I, et al (2001). Induction of pRb degradation by the human papillomavirus type $16 \mathrm{E} 7$ protein is essential to efficiently overcome p16INK4a-imposed G1 cell cycle Arrest. J Virol, 75, 4705-12.

Iaconis L, Hyjek E, Ellenson LH, et al (2007). p16 and Ki-67 immunostaining in atypical immature squamous metaplasia of the uterine cervix: correlation with human papillomavirus detection. Arch Pathol Lab Med, 131, 1343-9.

Katki HA, Gage JC, Schiffman M, et al (2013). Follow-up testing post-colposcopy: five-year risk of CIN2+ after a colposcopic diagnosis of CIN1 or less. J Low Genit Tract Dis, 17, 69-77.

Klaes R, Friedrich T, Spitkovsky D, et al (2001). Overexpression of p16INK4A as a specific marker for dysplastic and neoplastic epithelial cells of the cervix uteri. Int J Cancer, 92, 276-84.

Kurman RJ, Carcangiu ML, Herrington CS, et al (2014). WHO classification of tumours of female reproductive organs. pp 172.

Li J, Poi MJ, Tsai M-DJB (2011). Regulatory mechanisms of tumor suppressor P16INK4A and their relevance to cancer. Biochemistry, 50, 5566-82.

Liao GD, Sellors JW, Sun HK, et al (2014). p16INK4A immunohistochemical staining and predictive value for progression of cervical intraepithelial neoplasia grade 1: a prospective study in China. Int J Cancer, 134, 1715-24. 
Massad LS, Einstein MH, Huh WK, et al (2013). 2012 updated consensus guidelines for the management of abnormal cervical cancer screening tests and cancer precursors. J Low Genit Tract Dis, 121, 829-46.

Negri G, Vittadello F, Romano F, et al (2004). p16 INK4a expression and progression risk of low-grade intraepithelial neoplasia of the cervix uteri. Virchows Arch, 445, 616-20.

Pacchiarotti A, Ferrari F, Bellardini P, et al (2014). Prognostic value of p16-INK4A protein in women with negative or CIN1 histology result: A follow-up study. Int J Cancer, 134, 897-904.

Queriroz C, Silva T, Venancio AJPRP (2006). P16 expression as a potential prognostic marker in cervical pre-neoplastic and neoplatic lesion. Pathol Res Pract, 202, 77-83.

Razmpoosh M, Sansregret A, Oligny LL, et al (2014). Assessment of correlation between p16INK4a staining, specific subtype of human papillomavirus, and progression of LSIL/CIN1 lesions: first comparative study. Am J Clin Pathol, 142, 104-10.

Sagasta A, Castillo P, Saco A, et al (2016). p16 staining has limited value in predicting the outcome of histological low-grade squamous intraepithelial lesions of the cervix. Mod Pathol, 29, 51.

Sano T, Oyama T, Kashiwabara K, et al (1998). Expression status of p16 protein is associated with human papillomavirus oncogenic potential in cervical and genital lesions. $\mathrm{Am} \mathrm{J}$ Pathol, 153, 1741-8.

Sherris J, Herdman C, Elias CJWJoM (2001). Beyond our borders: cervical cancer in the developing world. West $J$ Med, 175, 231.

\section{(c) (i) (8)}

This work is licensed under a Creative Commons AttributionNon Commercial 4.0 International License. 